\title{
Passive immunotherapy, for the management of over 65 ages against the evolving fast spreading SARS CoV-2 variants infection
}

\author{
Jerard Seghatchian* \\ International Consultancy in Innovative Manufacturing and Quality/Safety of Blood-Derived Bioproducts, London, England, UK
}

\section{Introduction}

The Artificial Intelligence in the procedural, patterns and large data analyses have proven to be extremely useful in development/ deployment of vaccines and the related therapeutic modalities for the management of COVID infection [1-3]. No wonder, since WHO announce Coronavirus pandemic, many investigators have switched their research development studies to better understand the role played by various mode of immunotherapies including the coronavirus convalescent plasma $[\mathrm{CCP}]$ and the vaccine -induced neutralising antibodies for the prevention and management of various abnormalities associated with the Coronavirus acute respiratory diseases, as highlighted previously as the work in progress [4-10]. Clearly the 2021 is considered to be testing times for many of us, as CoV-2 with its evolving variants, is outwitting all of us and we must remain vigilante and committed to support both our community and the health care systems, to save some lives.

This commentary aimed to provide an update on the current status of passive immunotherapy, using the two currently approved vaccines that appear to provide a balance amount of CoV-2 neutralising antibodies, after 3-4 weeks to individuals in need for either preventative or therapeutic intervention. Attempts are also made, in particular, exploring the values of one single dose [instead of the two shots] protocol that has being recently introduced in the UK to overcome the shortage of vaccines delivery and distribution. The good news are: Firstly the UK Government took the boldest mass vaccination programme, by creating some groups of volunteers, helping in the spirit of oneness, in speedy effective mass vaccination scheme, a true UK- wide national efforts to cheer all us even as in 68 days 60 million jabs are given to most four high priority vulnerable populations that constitute $80-90 \%$ of death from Coronavirus. Secondly the preliminary data gathered so far indicate that even the first dose of two types of the given vaccines, have achieved $67 \%$ effectiveness and the infection rate is coming down rapidly by combination of vaccine therapy with partial lock down strategy. Nevertheless, long covid with mild and moderate symptoms appear to be not effected by these vaccines and we must focus on targeting the most virulent $\mathrm{CoV}-2$ variants and vaccines for children that are the topics of the current focuss. Moreover we should at least keep school staffs vaccinated and children apart as an urgent plans of action, as new school terms are starting soon, with a cautionary note the benefit versus the risk might be slightly reduced in the younger ages.

Fortunately, new vaccines involving also $\mathrm{N}$ protein in addition to spike protein is developing with the hope of enhancing higher levels of antibodies generation and immune $\mathrm{T}$ - cell responses. Moreover, newer vaccines to other genetically mutated are progressing to optimise the current state of the art of targeting the newer variants that we are doubtful the existing virus will be sufficiently effective. Moreover, it should be noted while vaccines are bringing slowly the hospitalisation levels down but not yet fully effective to reduce the transmission rate of the infection, in particular in view of fast spreading variants. Hence international collaborative efforts are required to tame infection, even with creating the requirement for travel passport and plenty of other travel restrictions as the race between targeted vaccine development and deployment using some modern and effective armatures must remain on agenda to survive the rate of viral mutation, until we bring down fully the rate of this infection with the expected human ingenuity.

\section{The current position of vaccines deployment in the UK}

In the context of where we are now and where we are going, while globally 9 candidate vaccines are in various stages of phase III safety/ efficacy trials for approval, as described by this author previously $[9,10]$ but only two well established manufacturers [Pfizer and AstraZeneca] have shown the capability to deal with the enormous demands, in view of the pandemic nature of this infection, as the shortage of the vaccines remains as the bottleneck of mass vaccination, in view of the shortage of viral vector, as being the case for the new Janssen and others vaccines. This is of particular relevance, as AstraZeneca had no choice that cut even the promised supply timely, which created enormous problematic concerns, in particular in European countries that were too slow in securing their supply well in advance as the UK and USA. No wander that The African Vaccine Acquisition Trust [ AVAT] has already entered in the advanced purchased agreement for 220 million doses on Janssen COVID vaccine. On the other hands Pfizer like some other producers, come to special deals to provide large scale supply to some countries, if they could get access the results as a joint venture, for example the mass vaccination with the greatest success in Israel and the planned Novavax, protein-based vaccine, in Australia, etc. [9]. Moreover, the evolving SARS CoV-2 variants, that spreading much faster than the original strains also enhanced the need for massive vaccination. This led to the current UK strategy, for delaying the second dose delivery of both vaccines from 3 weeks to 3

${ }^{\star}$ Correspondence to: Jerard Seghatchian, International Consultancy in Innovative Manufacturing and Quality/Safety of Blood-Derived Bioproducts, London, England, UK, E-mail jseghatchian@btopenworld.com

Received: February 17, 2021; Accepted: March 31, 2021; Published: April 05 2021 
months delivery scheme, to make the existing available vaccine supply to larger numbers of the four high priority groups.

\section{Conceptual acceptance of single JAB strategy}

It is noteworthy to highlight that the single jab strategy has never been tried before and also overriding the Pfizer approval protocol of 3-4weeks, but the WHO recently approved this protocol for the oxford vaccine, despite previously recommending an intermediate duration up to 6 weeks interval for the Pfizer vaccine, on the basis the best available evidence. However, in support of the UK decision it should be mentioned that the one dose of Pfizer/BioNTech's COVID-19 vaccine is $51 \%$ effective at preventing both symptomatic and asymptomatic SARS-CoV-2 infection and this level of efficacy meets the WHO acceptance criteria for the useful vaccine to be equal or over $50 \%$ efficacy. This concept is also supported by a new trial on Moderna' vaccine, using the same vaccination principle, than the Pfizer vaccine, indicating that that a single dose is effective after 2 months of vaccination. Hence single jab strategy based on available data could allow us to persue with a greater speed the global mass vaccination programme in the UK, later in the year, though at least 5 types vaccines including the Moderna, Novavax and even Johnson and Johnson, based of single dose principle, all under current evaluation by the $\mathrm{UK}$ as the global collaborative efforts, as these vaccines having some differing characteristic property in design and applications, as in the Moderna technology the mRNA of the spike proteins is encapsulated in a lipid capsule facilitating its storage and distribution at -20 freezer, in contrast to the Pfizer technology the vaccines need to be stored and transported at ultracold well below -70, making it operationally more difficult in some country with poorer infrastructures and the Oxford vaccine only required storage in cold room or fridges, so are new generation of vaccines, making them operationally simpler to control with reliability. The detail characteristic properties of other candidate vaccines are highlighted before [9]. Nevertheless, it should be point out that need for the second dose, as booster to optimise duration of their effectiveness with reliability and consistency cannot be denied.

\section{Current concerns in delaying the second JABS}

The delay the second vaccine dose for all the approved vaccines, is becoming as a matter of concerns in regard to optimisation of the immunity in individuals who have been vaccinated or has been exposed the virus and have already some measurable levels of high affinity antibodies in circulation. This is of particular relevance as the standard used in the various manufacturer's trials on vaccines, was based on the relative values on the level of antibodies seen in the convalescent plasma, at the time of studies, hence enhanced levels neutralsing antibodies remains to be clarified inline with the current opinions:

a] whether one or two doses of vaccine are required in people who have already been infected with SARS-CoV-2? As the one shot is probably sufficient, as there is some level of antibodies against the virus exist in people who were already infected and with one shot, they basically develop the level of antibodies that most people get with two shots of vaccine; b] Is the infection has primed the immune system and the on shot vaccine is effectively boosting those existing immune responses? It is clear that immunologically, infection generates immune responses, [ not just B-cell but also T-cell memory response], and some level of innate immunity but again there is enormous recipients' variability, but just one shot of vaccines or exposure to infection would be boosting immune responses to the point where they have antibody levels that physiologically are considered to be optimal for protection.
Even if you had mild disease such asymptomatic infection, just one shot appears to be able to bring the antibody levels up high enough to protect them against reinfection without vaccination; c] Do we need to correlate of protection against what antibody level is required as some people will cross the threshold of antibodies needed for protection right from start, with high efficacy vaccine and others who already had COVID might have more pronounced reactions to the second vaccine dose? This will be rather worrying, particularly in view of the very narrow window of time in between the first and second shots that if they are making a robust antibody response, the second shot might result in an even stronger reaction; d] Would these booster shots be advisable for people who have recovered from COVID? In fact, some vaccine companies are currently thinking about giving a third shot in an effort to boost immune responses and address the virus variants that are circulating, which is something that will need to be considered carefully for people who have already had COVID. Perhaps it would make sense to wait 3 months to get the booster if their antibody levels are waning; e] Given the limited vaccine supply, would it make more sense from a public health perspective to instead allocate these second doses to vaccinate more people? Hence, we could conserve that second dose and give it to people who really need it or using as vaccine boosters to address the viral variants, against which some existing vaccines seem to be slightly less effective requiring more 10 times antibodies to be effective.

\section{Future perspectives}

Clearly the deployment of existing COVID-19 vaccines, and the ways to optimize distribution of limited supply, the viral loads of some variants that continue to spread globally, and new approaches to vaccination that may offer protection more broadly against existing and emerging coronaviruses remains full of uncertainly and rising some pertinent questions that remain to be answered, despite numerous positive news in the pipeline:

i] A single dose of COVID-19 vaccine in healthcare workers who have already been infected with SARS-CoV-2 provides evidence that antibody responses to the first vaccine dose in individuals with preexisting immunity to SARS-CoV-2 is either equivalent to or better than that found in individuals who receive two doses of vaccine; ii] The B.1.1.7 SARS-CoV-2 variant that was first identified in the UK is of major concern for existing COVID-19 vaccines as this variant while is more transmissible, but it may also cause more severe disease however new trials on the approved Moderna's mRNA vaccine demonstrates reduced but still substantial neutralization of the B.1.351 SARS-CoV-2 variant that was first identified in South Africa; iv] Nanoparticle technologies in developing vaccines that can protect against multiple strains of coronavirus and this could be a potential strategy to protecting against SARS-CoV-2 variant strains and other emerging zoonotic coronaviruses; v] Meanwhile some studies are in progress examining how the timing of the booster dose of the AstraZeneca vaccine impacts its efficacy.

\section{Conclusion}

To sum up while the debate on the values of one versus two shot continues, despite the great success of the Israeli studies on over 55 old using two shots Pfizer vaccine and in view of the great number of uncertainties on the one shot protocol, we need to prioritise the balancing act between the speed of more dangerous viral development and the rate of global vaccination in this pandemic. Even considering that the current vaccines will be successful, we are still in need of more 
tools and novel approaches to effectively fight back the evolving SARS$\mathrm{CoV}-2$ variants contagion, by teaming up with prominent scientists and institutions from around the world to help find novel ways to protect people from SARS-CoV-2 infective attacks.

One of the ground breaking methods to prevent COVID-19 infection is using neutralsing polyclonal antibodies against the existing and emerging SARS-CoV-2 variants emerging SARS-CoV-2 variants [such as the fast spreading Kent- UK and the young South African strains] that can easily be obtained from some CCP mini-pool or from successfully vaccinationated mini pool of some good responders, to directly protect as a first line of defence against COVID.

The many advantages of this approach include to obtain purified neutralising antibody concentrate by affinity adoption column from the above mentioned mini pool free from the potentially infectioninduced various abnormalities, autoantibodies, residual viral infection and cytokines hence rapidly support the mass vaccination as a passive immunotherapy to catch the virus before it enters or exits the body by immobilizing SARS-CoV-2 and inhibiting viral binding to the ACE-2 receptor. This approach has been successfully used for immunotherapies to fight Influenza, and Ebola crises as the active therapeutic agents. The proven versatility of the immunotherapies offers interesting new opportunities as a method to prevent infections. The beauty of such a hyperconcentrate is that they are obtained from a mini pool from the real time covering all variants with some superior properties such as chemical stability, high affinity to a broad spectrum of epitopes, low immunogenicity, the ease of production and being promptly effective in an infection relevant SARS-CoV-2 virus model as compared to Vaccine therapy to generate high levels of antibody in 3-4 weeks in good responders.

Nevertheless we must always take into consideration crises and chaos observed in mental health and the socio- economic and in the physical wellbeing of the life that we are used too. Hence it remains essential that we comply closely and adhere to all restriction without dropping our guard to early as this virus leaned to mutate to escape our current mode of protective, in particular if given some chance to develop more dangerous variants without some continual surveillance and perseverance to stop its fast growth. These remind us again to recalibrate our thinking about how to approach the pandemic virus and shift our focus from the goal of herd immunity against transmission to the protection of all at risk individuals in population against severe disease that is the global aim of the AstraZenika virus of the world.

\section{References}

1. Seghatchian J (2020) Editorial: An introductory commentary on the use of artificial intelligence, machine learning and TQM, as novel computational tools in big data patterns or procedural analysis, in transfusion medicine. Transfus Apher Sci 59: 102985. [Crossref]

2. D, Alessandro A. Benford's law and metabolomics: a tale of numbers and blood. Transfus Apher Sci 59: 103019 [Crossref]

3. Sniecinski I, Seghatchian J (2018) Artificial Intelligence: A Joint Narrative on Potential Use in Pediatric Stem and Immune Cell Therapies and Regenerative Medicine. Transfus Apher Sci 57: 422-424. [Crossref]

4. Seghatchian J, Acker JP, Putter JS (2020) Update on newer approaches to prevent or treat COVID-19 infection: what we all need the most right now. Transfus Apher Sci 59: 102933 [Crossref]

5. Seghatchian J, Lanza F (2020) Convalescent plasma, an apheresis research project targeting and motivating the fully recovered COVID 19 patients: A rousing message of clinical benefit to both donors and recipients alike. Transfus Apher Sci 59: 102794 [Crossref]

6. Putter JS, Seghatchian J (2020) An update on COVID-19 infection control measures, plasma-based therapeutics, corticosteroid pharmacotherapy and vaccine research. Transfus Apher Sci 59: 102934. [Crossref]

7. Amiral J, Vissac AM, Seghatchian J (2020) COVID-19, induced activation of hemostasis, and immune reactions: Can an auto-immune reaction contribute to the delayed severe complications observed in some patients. Transfus Apher Sci 59: 102804 [Crossref]

8. Lanza F, Seghatchian J (2020) An overview of current position on cell therapy in transfusion medicine: from fictional promises to factual and perspectives from red cell substitution to stem cell therapy. Transfus Apher Sci 59: 102940 [Crossref]

9. Seghatchian J (2021) New generation of vaccines and convalescent plasma therapy for the management of CoV-2, in the global pandemic. Trans Apher Sci 2021: 103064.

10. Seghatchian J (2021) Commentary: Fact and challenges on global deployment of vaccines for the Immunotherapy of the evolving SARRS CoV-2 variants from the UK perspectives. Trends in transplant 14: 1-4.

Copyright: (C2021 Seghatchian J. This is an open-access article distributed under the terms of the Creative Commons Attribution License, which permits unrestricted use, distribution, and reproduction in any medium, provided the original author and source are credited. 